\title{
Visualization of the electron-solid interaction volume in dielectric materials under variable pressure conditions
}

\author{
Benjamin D. Myers* and Vinayak P. Dravid*\#
}

* NUANCE Center and Department of Materials Science and Engineering, Northwestern University, 2220 Campus Drive, Evanston, IL 60208

\# International Institute for Nanotechnology, Northwestern University, 2145 Sheridan Road, Evanston, IL 60208

The Variable Pressure Scanning Electron Microscope (VPSEM) has become a valuable tool for a number of applications in imaging and microanalysis of electrically insulating samples [1]. The environmental gas in the VPSEM chamber is ionized upon electron impact and the effects of surface charge on secondary electron (SE) imaging are minimized through the action of positive gas ions. There has been a significant amount of work to investigate the impact of the chamber gas on primary beam scattering and the charge-balance mechanisms as these questions have implications for applications in microanalysis, metrology and electron beam induced deposition [2]. Recently, we have applied the charge-balance mechanism of the VPSEM to the fabrication of nanometer-scale features on insulating substrates with a technique called variable pressure electron beam lithography (VP-eBL) [3-4]. The characterization of this process relies on the use of cross-sectional polymethyl methacrylate (PMMA) samples to visualize the shape and size of the electron interaction under varying exposure conditions. We present a general method for fabrication of cross-sectional PMMA samples and propose the extension of their use to study the general mechanisms of primary beam scattering and charging in the VPSEM.

The sample preparation process is outlined in Figure 1 and begins with spin coating clean, (100) oriented, lightly doped (>8 ohms-cm) silicon wafers with 950 PMMA (9\% solids in anisole) at a speed of 2000RPM to achieve a uniform film with thickness exceeding $1 \mu \mathrm{m}$. After spin coating, the silicon wafer was cleaved into rectangular fragments and a pre-crack was scribed normal to the edge of the fragment using a diamond stylus and a straight edge. Electron beam exposure was carried out in a FEI Quanta 600F ESEM with an integrated NPGS lithography system under varying conditions (accelerating voltage, chamber pressure, etc.). After exposure, the samples were developed in 3:1 mixture of isopropanol and methyl isobutyl ketone for various lengths of time. The developed samples and two pairs of tweezers were then immersed in liquid nitrogen. The samples were cleaved by placing the wafer on a fulcrum (e.g. paper clip) aligned with the pre-crack and pressing down on either side with the tweezers. The samples were then sputter coated with a 5-6 nm film of $\mathrm{Au} / \mathrm{Pd}$ prior to imaging in a high-resolution LEO Gemini 1525 SEM.

The resulting cross sections provide fracture surfaces through the exposed PMMA areas without distortion or smearing of the PMMA. A relatively low magnification image of one such cross section is shown in Figure 2. Analysis of the cross sections shows decreasing trench size as a function of increasing gas pressure due to primary beam scattering in the chamber gas. A modification of the trench shape can also be visualized with increasing gas pressure, indicating a modification of the sub-surface space charge distribution. By increasing the developing time or developer concentration, regions of lower electron dose are exposed. A collection of cross section images are shown in Figure 3 and show qualitatively that primary electron scattering, while 
responsible for attenuation of the primary beam, results in a broad distribution of electrons, and no appreciable change in the probe size. In combination with a rigorous Monte Carlo or analytical model, these cross sections could be used to determine the distribution of primary electrons in close proximity (e.g. within 1 micron) to the electron beam as well as dynamic charging effects.

References

[1] GD Danilatos, Adv. Electron. Electron Phys. 71 (1988) 109.

[2] BL Thiel and M Toth, J. Appl. Phys. 97 (2005) 051101.

[3] BD Myers and VP Dravid, Microsc. Microanal. 11 (suppl. 2) (2005) 392.

[4] BD Myers and VP Dravid, (submitted to Nano Lett.).

[5] This research is partially supported by NUANCE staff development program and NU-NSEC.
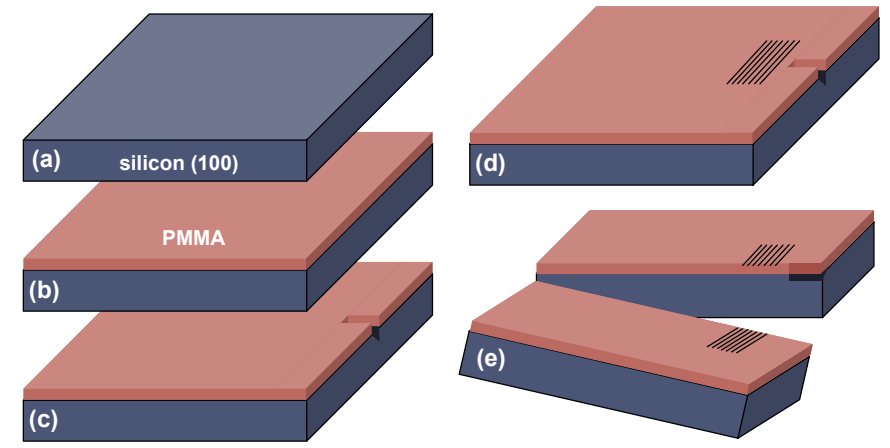

FIG.1. Schematic representation of sample preparation: a) clean silicon substrate b) spin coat with PMMA, c) scribe pre-crack along cleavage direction, d) electron beam patterning at end of precrack e) cleavage under liquid nitrogen

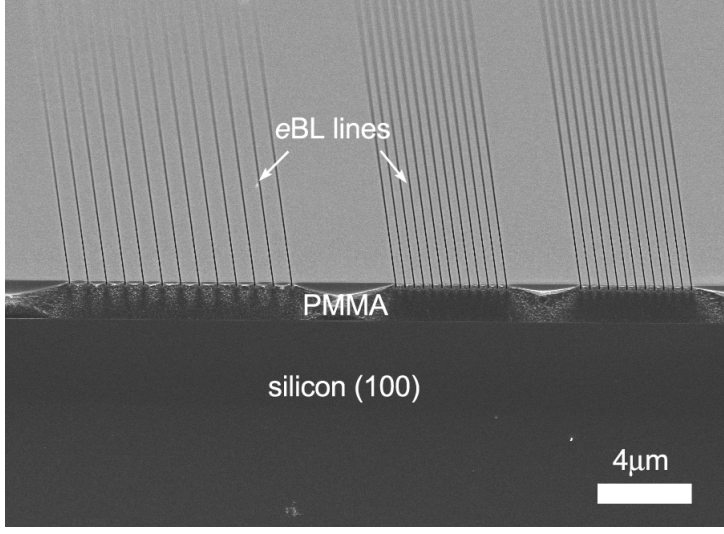

FIG. 2. SEM image of $\mathrm{Au} / \mathrm{Pd}$ coated cross sectional sample showing lines of different spacing (400 and 800nm)


FIG. 3. Cross sectional samples prepared under different conditions: (a-c) $15 \mathrm{kV}, 1.0 \mathrm{nC} / \mathrm{cm}$ dose, $120 \mathrm{sec}$. develop and a) high vacuum, b) 0.5 Torr and c) 1 Torr water vapor; (d-f) 5kV, $0.5 \mathrm{nC} / \mathrm{cm}$ dose, $120 \mathrm{sec}$. develop and d) high vacuum, e) 0.5 Torr and f) 1 Torr water vapor. 